\title{
Whistler oscillitons revisited: the role of charge neutrality?
}

\author{
F. Verheest ${ }^{1,2}$, T. Cattaert ${ }^{1}$, E. Dubinin ${ }^{3}$, K. Sauer ${ }^{3}$, and J. F. McKenzie ${ }^{2,3}$ \\ ${ }^{1}$ Sterrenkundig Observatorium, Universiteit Gent, Krijgslaan 281, 9000 Gent, Belgium \\ ${ }^{2}$ School of Physics (Howard College Campus), University of KwaZulu-Natal, Durban 4041, South Africa \\ ${ }^{3}$ Max-Planck-Institut für Aeronomie, D-37191 Katlenburg-Lindau, Germany
}

Received: 6 August 2004 - Revised: 11 October 2004 - Accepted: 15 October 2004 - Published: 20 October 2004

Part of Special Issue "Advances in space environment turbulence"

\begin{abstract}
When studying transverse modes propagating parallel to a static magnetic field, an apparent contradiction arises between the weakly nonlinear results obtained from the derivative nonlinear Schrödinger equation, predicting envelope solitons (where the amplitude is stationary in the wave frame, but the phase is not), and recent results for whistler oscillitons, indicating that really stationary structures of large amplitude are possible. Revisiting this problem in the fluid dynamic approach, care has been taken not to introduce charge neutrality from the outset, because this not only neglects electric stresses compared to magnetic stresses, which is reasonable, but could also imply from Poisson's equation a vanishing of the wave electric field. Nevertheless, the fixed points of the remaining equations are the same, whether charge neutrality is assumed from the outset or not, so that the solitary wave solutions at not too large amplitudes will be very similar. This is borne out by numerical simulations of the solutions under the two hypotheses, showing that the lack of correspondence with the DNLS envelope solitons indicates the limitations of the reductive perturbation approach, and is not a consequence of assuming charge neutrality.
\end{abstract}

\section{Introduction}

In recent observations of space plasmas strongly nonlinear wave phenomena have been seen. For electrostatic modes the theoretical large amplitude explanations based on the Sagdeev or on the fluid dynamic treatment tally well both with observations and with earlier knowledge based on weakly nonlinear approaches, where the reductive perturbation techniques lead to the Korteweg-de Vries (KdV) or modified $\mathrm{KdV}(\mathrm{mKdV})$ equations as the typical paradigm. Similar conclusions can be drawn for modes that propagate obliquely or perpendicularly with respect to an external magnetic field.

Correspondence to: F. Verheest

(Frank.Verheest@UGent.be)
By contrast, a large amplitude theory for parallel electromagnetic modes is either lacking or, when such large amplitude stationary structures are explained in terms of oscillitons, the latter approach does not agree with what is known from the weak amplitude treatments. Indeed, the reductive perturbation analysis leads for parallel electromagnetic modes in ordinary plasmas (where the ion and electron masses are very different) to the derivative nonlinear Schrödinger (DNLS) equation as the typical nonlinear evolution equation. As is the case with the $\mathrm{KdV}$ or $\mathrm{mKdV}$ equations, the DNLS equation is a perfectly integrable nonlinear evolution equation, with an infinite number of conserved densities or first integrals, and hence with $N$-soliton solutions for every integer $N$.

Nevertheless, the DNLS solitons are not completely stationary structures but envelope solitons, where the amplitude is stationary in the wave frame, but the phase is not, the latter showing a slow increase with time. Recent results (Dubinin et al., 2003, 2004) concerning parallel propagating electromagnetic oscillitons indicate that really stationary nonlinear solutions can exist in ordinary plasmas for these modes. Although the oscillitons (Sauer et al., 2001, 2002, 2003; Sauer and Dubinin, 2003; Dubinin et al., 2003, 2004) superficially look like envelope solitons, the phase is stationary for the former but not for the latter. Given the earlier weakly nonlinear results, we would, however, not expect such truly stationary solutions to be possible, unless the DNLS equation is not the appropriate nonlinear evolution equation for oscillitons of weaker amplitude.

This conundrum disappears in the study of similar waves in electron-positron plasmas (Verheest and Cattaert, 2004), because the positive and negative particles have the same mass and opposite charges. Such a symmetry equalizes the scales and the reductive perturbation approach generates a vector $\mathrm{mKdV}$-type equation, which is nonintegrable except for linearly polarized modes (Verheest, 1996), and then stationary solitons are possible, of both large and small amplitudes. 
Because of the mentioned differences between the DNLS envelope solitons and the whistler oscillitons, we revisit the description of parallel propagating electromagnetic modes. As in earlier studies (Sauer et al., 2001, 2002, 2003; Sauer and Dubinin, 2003; Dubinin et al., 2003, 2004) we will use the fluid dynamic approach of studying nonlinear stationary structures in their own reference frame, but without imposing charge neutrality a priori (the plasma approximation), in an attempt to clarify the possible role played by that assumption.

A number of first integrals can be derived and this yields a corresponding reduction in the number of equations left to solve. As we will indicate after a careful analysis of the existing framework and of a discussion of possible solutions in terms of fixed points of the remaining differential equations, it is not the assumption of charge neutrality that is responsible for the discrepancy with the DNLS results. We offer some suggestions as to the direction(s) in which to extend our present understanding of these interesting nonlinear wave types, of undoubted heliospheric importance.

\section{Basic equations and multispecies invariants}

We repeat some of the methodology from an earlier paper on solitary structures in electron-proton plasmas (Verheest and Cattaert, 2004), where the equal masses gave rise to a mixing of all scales and hence to exceptional results. The x-axis of the reference system is taken along the direction of the external magnetic field $\mathbf{B}_{0}=B_{0} \mathbf{e}_{x}$, and we look at solitary wave structures propagating along the static field, so that the only spatial coordinate is $x$. In the frame moving with the nonlinear structure, all plasma species have an undisturbed reference speed $V$ along the $\mathrm{x}$-axis at $x=-\infty$. The standard set of cold multispecies plasma equations includes per species the continuity and momentum equations,

$$
\begin{aligned}
& \frac{\partial n_{j}}{\partial t}+\frac{\partial}{\partial x}\left(n_{j} v_{j x}\right)=0, \\
& \frac{\partial \mathbf{v}_{j}}{\partial t}+v_{j x} \frac{\partial \mathbf{v}_{j}}{\partial x}=\frac{q_{j}}{m_{j}}\left(\mathbf{E}+\mathbf{v}_{j} \times \mathbf{B}\right),
\end{aligned}
$$

where $n_{j}$ and $\mathbf{v}_{j}$ refer to the number density and fluid velocity of each plasma species, with charge $q_{j}$ and mass $m_{j}$, while $\mathbf{E}$ and $\mathbf{B}$ are the electric and (total) magnetic fields, respectively. When we treat standard electron-ion plasmas, we will use the subscripts $j=e$ for the electrons and $j=i$ for the ions. To be able to still go to the positron limit, however, we will for the time being avoid using $m_{e} \ll m_{i}$. The system is closed by Maxwell's equations

$$
\begin{aligned}
& \mathbf{e}_{x} \times \frac{\partial \mathbf{E}}{\partial x}+\frac{\partial \mathbf{B}}{\partial t}=\mathbf{0}, \\
& \mathbf{e}_{x} \times \frac{\partial \mathbf{B}}{\partial x}=\frac{1}{c^{2}} \frac{\partial \mathbf{E}}{\partial t}+\mu_{0} \sum_{j} n_{j} q_{j} \mathbf{v}_{j}, \\
& \varepsilon_{0} \frac{\partial E_{x}}{\partial x}=\sum_{j} n_{j} q_{j}, \\
& \frac{\partial B_{x}}{\partial x}=0 .
\end{aligned}
$$

The last equation and the parallel component of the first show that $B_{x}=B_{0}$ is constant. Since in the wave frame there is no time variation for stationary nonlinear structures, some of these equations can easily be integrated in $x$, the sole independent variable remaining. We thus arrive from the continuity Eq. (1) at conservation of parallel (mass) flux per species,

$n_{j} v_{j x}=n_{j 0} V$.

Combining the equations of motion Eq. (2) with Maxwell's equations Eq. (3), four additional and distinct global integrals of motion arise,

$$
\begin{aligned}
& \sum_{j} n_{j 0} m_{j}\left(v_{j x}-V\right)+\frac{B_{\perp}^{2}}{2 \mu_{0} V}-\frac{\varepsilon_{0} E_{x}^{2}}{2 V}=0, \\
& \sum_{j} n_{j 0} m_{j} \mathbf{v}_{j \perp}=\frac{B_{0}}{\mu_{0} V} \mathbf{B}_{\perp}, \\
& \sum_{j} n_{j 0} m_{j}\left(v_{j x}^{2}+v_{j \perp}^{2}-V^{2}\right)=0, \\
& \sum_{j} \frac{n_{j 0} m_{j}^{2} v_{j \perp}^{2}}{q_{j}}=0,
\end{aligned}
$$

valid for general plasma compositions. For intermediate details of the derivation we refer to Verheest and Cattaert (2004), and remark that our multispecies invariants generalize the ones obtained by Dubinin et al. $(2003,2004)$ for standard electron-ion plasmas.

\section{Electron-ion plasmas}

We now specialize the results obtained so far to an electronion plasma, where $q_{e}=-e, q_{i}=+e$, and $n_{e 0}=n_{i 0}=n_{0}$ due to charge neutrality in equilibrium. Contrary to what is done in a recent description of whistler oscillitons (Dubinin et al., 2003), we will not yet assume that charge neutrality always holds, but will explore the consequences of that ansatz at a later stage.

\subsection{Discussion of first integrals}

The first integrals Eq. (4) and (5) become for electron-ion plasmas,

$$
\begin{aligned}
& n_{e} v_{e x}=n_{i} v_{i x}=n_{0} V, \\
& m_{e} v_{e x}+m_{i} v_{i x}+\frac{B_{\perp}^{2}}{2 \mu_{0} n_{0} V}-\frac{\varepsilon_{0} E_{x}^{2}}{2 n_{0} V}=\left(m_{e}+m_{i}\right) V, \\
& m_{e} \mathbf{v}_{e \perp}+m_{i} \mathbf{v}_{i \perp}=\frac{B_{0}}{\mu_{0} n_{0} V} \mathbf{B}_{\perp}, \\
& m_{e} v_{e x}^{2}+m_{i} v_{i x}^{2}+m_{e} v_{e \perp}^{2}+m_{i} v_{i \perp}^{2}=\left(m_{e}+m_{i}\right) V^{2}, \\
& m_{e}^{2} v_{e \perp}^{2}=m_{i}^{2} v_{i \perp}^{2} .
\end{aligned}
$$

This set of invariants amounts to 7 scalar algebraic relations between the 11 dependent variables $n_{e}, n_{i}, v_{e x}, v_{e y}, v_{e z}, v_{i x}$, $v_{i y}, v_{i z}, B_{y}, B_{z}$ and $E_{x}$. In principle we will need to use at most 4 differential equations to determine the system completely. 
Given that $v_{e \perp}^{2}=v_{e y}^{2}+v_{e z}^{2}, v_{i \perp}^{2}=v_{i y}^{2}+v_{i z}^{2}$ and $B_{\perp}^{2}=B_{y}^{2}+B_{z}^{2}$, we represent the three perpendicular vector variables in a polar decomposition through amplitudes and phases as

$$
\begin{aligned}
& \mathbf{v}_{e \perp}=v_{e \perp}\left(\mathbf{e}_{y} \cos \alpha_{e}+\mathbf{e}_{z} \sin \alpha_{e}\right), \\
& \mathbf{v}_{i \perp}=v_{i \perp}\left(\mathbf{e}_{y} \cos \alpha_{i}+\mathbf{e}_{z} \sin \alpha_{i}\right), \\
& \mathbf{B}_{\perp}=B_{\perp}\left(\mathbf{e}_{y} \cos \beta+\mathbf{e}_{z} \sin \beta\right),
\end{aligned}
$$

and replace thus $v_{e y}, v_{e z}, v_{i y}, v_{i z}, B_{y}$ and $B_{z}$ by three amplitudes $v_{e \perp}, v_{i \perp}, B_{\perp}$ and three phases $\alpha_{e}, \alpha_{i}, \beta$. After scalar multiplication of (8) by, respectively, $m_{e} \mathbf{v}_{e \perp}$ and $m_{i} \mathbf{v}_{i \perp}$, we see that due to Eq. (10) the 1.h.s. are equal, and so are the r.h.s.,

$m_{e} \mathbf{B}_{\perp} \cdot \mathbf{v}_{e \perp}=m_{i} \mathbf{B}_{\perp} \cdot \mathbf{v}_{i \perp}$

Keeping in mind that $m_{e} v_{e \perp}=m_{i} v_{i \perp}$, this is equivalent to

$$
\begin{aligned}
& \cos \left(\alpha_{e}-\beta\right)-\cos \left(\alpha_{i}-\beta\right) \\
& =2 \sin \left(\frac{\alpha_{i}+\alpha_{e}}{2}-\beta\right) \sin \left(\frac{\alpha_{i}-\alpha_{e}}{2}\right)=0 .
\end{aligned}
$$

In a similar vein we postmultiply Eq. (8) vectorially by $\mathbf{B}_{\perp}$ to obtain

$m_{e} \mathbf{v}_{e \perp} \times \mathbf{B}_{\perp}+m_{i} \mathbf{v}_{i \perp} \times \mathbf{B}_{\perp}=\mathbf{0}$,

from which it follows that

$$
\begin{aligned}
& \sin \left(\alpha_{i}-\beta\right)+\sin \left(\alpha_{e}-\beta\right) \\
& =2 \sin \left(\frac{\alpha_{i}+\alpha_{e}}{2}-\beta\right) \cos \left(\frac{\alpha_{i}-\alpha_{e}}{2}\right)=0
\end{aligned}
$$

Because $\sin \left[\left(\alpha_{i}-\alpha_{e}\right) / 2\right]$ and $\cos \left[\left(\alpha_{i}-\alpha_{e}\right) / 2\right]$ cannot vanish together, the combination of Eq. (13) and (15) only allows the possibility that

$\sin \left(\frac{\alpha_{i}+\alpha_{e}}{2}-\beta\right)=0$,

in other words, the phases are locked in the sense that

$\alpha_{i}+\alpha_{e}=2 \beta$

One can also multiply (8) scalarly by $\mathbf{B}_{\perp}$ and obtain

$B_{\perp}=\frac{2 \mu_{0} n_{0} V}{B_{0}} m_{i} v_{i \perp} \cos \left(\alpha_{i}-\beta\right)$.

Since phase differences like $\alpha_{i}-\beta$ or $\alpha_{i}-\alpha_{e}$ frequently occur, we introduce for later use $\varphi=2\left(\alpha_{i}-\beta\right)=\alpha_{i}-\alpha_{e}$. Other operations on Eq. (8) yield equivalent results, given the relation (17) between the phases.

Among the remaining first integrals, Eq. (9) can be combined with Eq. (10) to link perpendicular velocity moduli like $v_{i \perp}$ to the parallel velocities, yielding

$v_{i \perp}^{2}=\frac{m_{e}}{m_{i}}\left(V^{2}-\frac{m_{e} v_{e x}^{2}+m_{i} v_{i x}^{2}}{m_{i}+m_{e}}\right)$,

which we will use later on. Finally, squaring the relation (18) gives an expression for $B_{\perp}^{2}$ which can be used in Eq. (7) to express $E_{x}$ in terms of the parallel velocities and of $\varphi$, with the help of Eq. (19). This yields

$$
\begin{aligned}
E_{x}^{2}= & \frac{2 \mu_{0} n_{0}^{2} V^{2} m_{i} m_{e}}{\varepsilon_{0} B_{0}^{2}}\left(V^{2}-\frac{m_{e} v_{e x}^{2}+m_{i} v_{i x}^{2}}{m_{i}+m_{e}}\right)(1+\cos \varphi) \\
& +\frac{2 n_{0} V}{\varepsilon_{0}}\left[m_{e} v_{e x}+m_{i} v_{i x}-\left(m_{i}+m_{e}\right) V\right],
\end{aligned}
$$

which is, however, an unwieldy expression that cannot readily be used to extract further restrictions on the admissible solitary wave solutions. We will therefore use a more indirect way of discussing possible scenarios.

\subsection{Equations of motion}

Here we decompose the perpendicular ion equation of motion

$m_{i} v_{i x} \frac{d \mathbf{v}_{i \perp}}{d x}=e B_{0} \mathbf{v}_{i \perp} \times \mathbf{e}_{x}+e v_{i x} \mathbf{e}_{x} \times \mathbf{B}_{\perp}$,

into amplitude and phase changes,

$$
\begin{aligned}
\frac{d v_{i \perp}}{d x} & =\frac{\mu_{0} n_{0} V e}{B_{0}} v_{i \perp} \sin \varphi, \\
\frac{d \alpha_{i}}{d x} & =-\frac{e B_{0}}{n_{0} V} \frac{n_{i}}{m_{i}}+\frac{\mu_{0} n_{0} V e}{B_{0}}(1+\cos \varphi) .
\end{aligned}
$$

There are analogous and sometimes, like for the electron amplitude equation, equivalent results for the electrons, so that

$$
\begin{aligned}
\frac{d v_{e \perp}}{d x} & =\frac{\mu_{0} n_{0} V e}{B_{0}} v_{e \perp} \sin \varphi, \\
\frac{d \alpha_{e}}{d x} & =\frac{e B_{0}}{n_{0} V} \frac{n_{e}}{m_{e}}-\frac{\mu_{0} n_{0} V e}{B_{0}}(1+\cos \varphi) .
\end{aligned}
$$

Results are expressed in the ion quantities where possible. Using Eq. (17) it follows that

$$
\frac{d \varphi}{d x}=-\frac{e B_{0}}{n_{0} V}\left(\frac{n_{i}}{m_{i}}+\frac{n_{e}}{m_{e}}\right)+\frac{2 \mu_{0} n_{0} V e}{B_{0}}(1+\cos \varphi) .
$$

In the above expressions, one can when needed substitute $n_{i}$ and $n_{e}$ in terms of the corresponding parallel velocities $v_{i x}$ and $v_{e x}$ with the help of mass flux conservation Eq. (6).

Rearranging the parallel equations of motion is slightly more complicated, and it is here that some of the possible implications of assuming quasi charge neutrality will manifest themselves. We start the discussion from

$\begin{aligned} v_{i x} \frac{d v_{i x}}{d x} & =\frac{e}{m_{i}} E_{x}-\frac{\mu_{0} n_{0} V e}{B_{0}} v_{i \perp}^{2} \sin \varphi, \\ v_{e x} \frac{d v_{e x}}{d x} & =-\frac{e}{m_{e}} E_{x}-\frac{\mu_{0} n_{0} V e}{B_{0}} v_{e \perp}^{2} \sin \varphi .\end{aligned}$

Multiplying these equations by $m_{i}^{2}$ and $m_{e}^{2}$, respectively, and subtracting the resulting expressions allows us to write the parallel electric field in an indirect way as

$E_{x}=\frac{1}{e\left(m_{i}+m_{e}\right)}\left(m_{i}^{2} v_{i x} \frac{d v_{i x}}{d x}-m_{e}^{2} v_{e x} \frac{d v_{e x}}{d x}\right)$. 
When the derivative is taken of $E_{x}^{2}$ as given in Eq. (20), and $E_{x}$ (but not $d E_{x} / d x$ ) is replaced by the expression (26), one finds after some interesting algebra the equivalent of Poisson's equation. This is not altogether surprising, since Eq. (7) was obtained by using i.a. Poisson's equation.

Suppose now that we assume charge neutrality, $n_{i}=n_{e}=n$, then mass conservation Eq. (6) leads to equal parallel velocities for ions and electrons, $v_{i x}=v_{e x}=v_{x}$. Of course, charge neutrality implies from Poisson's equation that $\varepsilon_{0} d E_{x} / d x=0$. In order to admit a nontrivial $E_{x}$ but yet consider quasi charge neutrality, we formally take the limit that $\varepsilon_{0} \rightarrow 0$, i.e. using a strictly nonrelativistic treatment, whereby the electric stresses can be neglected in comparison to the magnetic stresses. We rewrite the expression (26) for $E_{x}$ for equal parallel ion and electron velocities as

$E_{x}=\frac{m_{i}-m_{e}}{e} v_{x} \frac{d v_{x}}{d x}$,

and see that $E_{x}$ vanishes when $m_{i}=m_{e}$, in an electronpositron plasma (Verheest and Cattaert, 2004). Thus, overall charge neutrality and equal parallel velocities can consistently be assumed when dealing with an electron-positron plasma, and in ordinary electron-ion plasmas the electric effects remain small, as long as we deal with nonrelativistic phenomena under the plasma approximation.

\subsection{Fixed points}

To continue our general discussion and in analogy with the earlier treatment by Dubinin et al. (2003) for the charge neutral case, we are looking for stationary solutions, with the disturbance vanishing at infinity, so that the state at infinity must be an equilibrium point. Hence we now turn to the fixed points for the set of equations consisting of Poisson's equation from Eq. (3), the phase Eq. (24) and the two Eq. (25), where $v_{i \perp}^{2}$ has to be replaced by Eq. (19) and $v_{e \perp}^{2}$ similarly by $m_{i}^{2} v_{i \perp}^{2} / m_{e}^{2}$,

$$
\begin{aligned}
\frac{d E_{x}}{d x} & =\frac{e n_{0} V}{\varepsilon_{0} v_{i x}}-\frac{e n_{0} V}{\varepsilon_{0} v_{e x}}, \\
\frac{d \varphi}{d x} & =-e B_{0}\left(\frac{1}{m_{i} v_{i x}}+\frac{1}{m_{e} v_{e x}}\right) \\
& +\frac{2 \mu_{0} n_{0} V e}{B_{0}}(1+\cos \varphi), \\
\frac{d v_{i x}}{d x} & =\frac{e E_{x}}{m_{i} v_{i x}} \\
& -\frac{\mu_{0} n_{0} V e m_{e}}{B_{0} m_{i} v_{i x}}\left(V^{2}-\frac{m_{e} v_{e x}^{2}+m_{i} v_{i x}^{2}}{m_{i}+m_{e}}\right) \sin \varphi, \\
\frac{d v_{e x}}{d x} & =-\frac{e E_{x}}{m_{e} v_{e x}} \\
& -\frac{\mu_{0} n_{0} V e m_{i}}{B_{0} m_{e} v_{e x}}\left(V^{2}-\frac{m_{e} v_{e x}^{2}+m_{i} v_{i x}^{2}}{m_{i}+m_{e}}\right) \sin \varphi .
\end{aligned}
$$

Such a set of equations remaining to be solved is not unique, and other choices could have been made instead, but the present set has the advantage of expressing the derivatives of four variables in terms of these same variables. Strictly speaking, one of the four differential equations in (28) could have been replaced by information coming from the invariant (20). At the end one would still need to solve one of the other phase equations in Eq. (22) or (23) for $\alpha_{i}$ or $\alpha_{e}$, respectively, in order to determine all variables completely.

The conditions for the fixed points are

$$
\begin{aligned}
& v_{e x}=v_{i x}, \\
& \cos \varphi=\frac{B_{0}^{2}}{2 \mu_{0} n_{0} V}\left(\frac{1}{m_{i} v_{i x}}+\frac{1}{m_{e} v_{e x}}\right)-1, \\
& E_{x}=0, \\
& \left(V^{2}-\frac{m_{e} v_{e x}^{2}+m_{i} v_{i x}^{2}}{m_{i}+m_{e}}\right) \sin \varphi=0 .
\end{aligned}
$$

This shows that in a fixed point $E_{x}$ vanishes, not because Poisson's law has been used, but resulting from the two parallel equations of motion. Also, the parallel velocities are equal, implying charge neutrality $n_{e}=n_{i}$.

Mathematically speaking, there are two separate fixed points, one with two possibilities for the phase $\varphi$. First comes $v_{e x}=v_{i x}=V$, implying from Eq. (19) that $v_{i \perp}=v_{e \perp}=0$, and from Eq. (18) also that $B_{\perp}=0$. The phase is given through

$$
\cos \varphi=\frac{B_{0}^{2}}{2 \mu_{0} n_{0} V^{2}}\left(\frac{1}{m_{i}}+\frac{1}{m_{e}}\right)-1,
$$

and there are two opposite values for $\varphi$. This fixed point agrees with the first one obtained by Dubinin et al. (2003), as we will show below, when their normalized variables are used and $m_{e} \ll m_{i}$ is assumed.

The other possibility is that we suppose $v_{e x}=v_{i x} \neq V$, which leads to $\sin \varphi=0$ and hence $\cos \varphi=1$. Consequently, Eq. (29) then yields

$v_{e x}=v_{i x}=\frac{B_{0}^{2}}{4 \mu_{0} n_{0} V}\left(\frac{1}{m_{i}}+\frac{1}{m_{e}}\right)$.

It is readily seen that we recover the second fixed point discussed by Dubinin et al. (2003), again by going to their normalized variables and assuming that $m_{e} \ll m_{i}$.

Indeed, assuming charge neutrality from the outset and rewriting in our fully dimensional notation the remaining set of two differential equations to be solved, equivalent to those discussed by Dubinin et al. (2003), the fixed points have now to be determined from

$$
\begin{aligned}
\frac{d \varphi}{d x} & =-\frac{e B_{0}}{v_{x}}\left(\frac{1}{m_{i}}+\frac{1}{m_{e}}\right)+\frac{2 \mu_{0} n_{0} V e}{B_{0}}(1+\cos \varphi), \\
\frac{d v_{x}}{d x} & =-\frac{\mu_{0} n_{0} V e}{B_{0} v_{x}}\left(V^{2}-v_{x}^{2}\right) \sin \varphi .
\end{aligned}
$$

The fixed points here are the solutions of

$$
\begin{aligned}
& \cos \varphi=\frac{B_{0}^{2}}{2 \mu_{0} n_{0} V v_{x}}\left(\frac{1}{m_{i}}+\frac{1}{m_{e}}\right)-1, \\
& \left(V^{2}-v_{x}^{2}\right) \sin \varphi=0,
\end{aligned}
$$



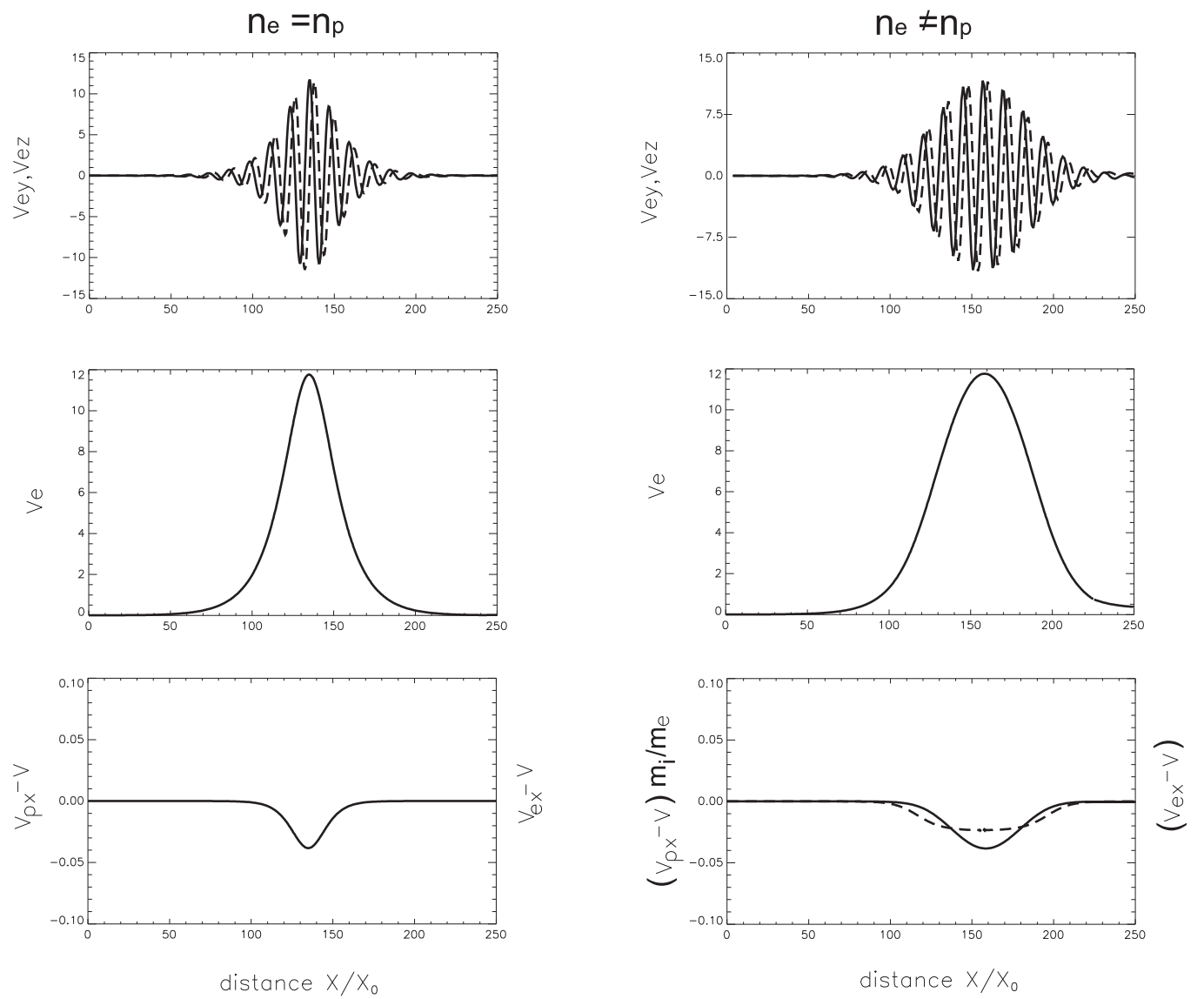

Fig. 1. The structure of oscillitons propagating with velocity $V=0.505 V_{A e}$ in an electron-proton plasma obtained under two different assumptions. The results shown in the left column are obtained assuming charge neutrality. The plots in the right column are obtained without this assumption $\left(\gamma=V_{A e}^{2} / c^{2}=0.01\right)$.

and correspond exactly to those derived in the full treatment.

It has to be remarked that the second fixed point is not accessible to the solitary wave solutions we are looking for, because the invariant (20) is not obeyed for the boundary conditions imposed. Nevertheless, the fixed points of the set Eq. (28) or (32) will help in discussing possible solutions of these equations in a phase space diagram. Since the fixed points are the same, it is reasonable to assume that the solitary wave solutions at not too large amplitudes will be very similar, whether quasi charge neutrality is used or not.

This is indeed borne out by numerical computations of the solutions of Eq. (28) and (32), as shown in Fig. 1. The left column in Fig. 1 represents the oscilliton structure obtained under the assumption of quasi-neutrality. The upper panel depicts the transverse electron velocity components $v_{e y}$ (full curve) and $v_{e z}$ (dashed curve), respectively. The middle panel shows the modulus of the transverse speed, $v_{e}=\left(v_{e y}^{2}+v_{e z}^{2}\right)^{1 / 2}$. In the bottom panel the longitudinal components of the electron (ion) speed $\left(v_{e x}=v_{i x}\right)$ is given, obtained from the integral of motion Eq. (7) while neglecting the term which describes the electric field stresses. All velocity values are normalized to the Alfvén speed $V_{A e}$ based on the electron mass density. The distance $x$ is normalized to the value $V_{A e} / \Omega_{e}$, where $\Omega_{e}$ is the (absolute value of) the electron gyrofrequency. The oscilliton speed itself is taken as $V=0.505 V_{A e}$. The right column represents the results of numerically solving for the oscilliton structure without assuming charge neutrality, for the same parameters. The amplitude of the oscilliton $v_{e}$ remains almost the same, although the width broadens. The longitudinal components of the velocity of the species are now different, providing for a nonvanishing electric field $E_{x}$. The protons are almost at rest $\left(\delta v_{p x} \simeq \delta v_{e x} m_{e} / m_{i}\right)$. This vindicates our investigation into the role of charge neutrality.

\section{Conclusions}

We were intrigued by the apparent contradiction between the DNLS results, which predict envelope solitons, where the amplitude is stationary in the wave frame, but the phase is not, and recent results (Dubinin et al., 2003) for parallel propagating electromagnetic oscillitons, which indicated that really stationary nonlinear structures of large amplitude and stationary phase exist. Given the weakly nonlinear reductive perturbation results, we would not expect such truly stationary structures to be possible. 
The fluid dynamic approach of studying nonlinear stationary structures in their own reference frame was then followed, but we did not introduce charge neutrality from the very beginning. However, the fixed points of the set of remaining differential equations to be solved turn out to be the same, whether charge neutrality is imposed from the beginning or not. It therefore is reasonable to assume that the solitary wave solutions, the oscillitons, will be very similar at not too large amplitudes, and this is indeed borne out by numerical computations of the solutions of the two sets of equations.

Hence we conclude that the discrepancies between the whistler oscillitons and the DNLS envelope solitons are not at all attributable to the plasma approximation of quasi charge neutrality, but indicate that the correct nonlinear evolution equation corresponding to oscillitons is not yet available, the derivation of which, moreover, is not obvious at all.

Acknowledgements. Interesting discussions with M. A. Hellberg, S. R. Pillay and P. K. Shukla are gratefully acknowledged. FV and TC thank the Fonds voor Wetenschappelijk Onderzoek (Vlaanderen) for a research grant. The four senior authors have greatly benefitted from the kind hospitality of the International Space Science Institute (Berne, Switzerland), where part of this work was discussed during a meeting of the International Team on "Nonlinear Plasma Waves - Solitons, Periodic Waves and Oscillitons in Diverse Space Plasma Environments". This work was also supported by a bilateral cooperation grant on "Physics of Dusty and Space Plasmas" between Flanders (Onderzoekfonds Universiteit Gent) and South Africa (National Research Foundation).

Edited by: T. Passot

Reviewed by: E. Mjolhus and R. A. Treumann

\section{References}

Dubinin, E., Sauer, K., and McKenzie, J. F.: Nonlinear stationary whistler waves and whistler solitons (oscillitons), Exact solutions, J. Plasma Phys., 69, 305-330, 2003.

Dubinin, E., Sauer, K., and McKenzie, J. F.: Nonlinear stationary waves and solitons in ion beam-plasma configuration, J. Geophys. Res., 109, A02208, 2004.

Sauer, K. and Dubinin, E.: Oscillitons and gyrating ions in a beamplasma system, Geophys. Res. Lett., 30, art.no. 2192, 2003.

Sauer, K., Dubinin, E., and McKenzie, J. F.: New type of soliton in bi-ion plasmas and possible implications, Geophys. Res. Lett., 28, 3589-3592, 2001.

Sauer, K., Dubinin, E., and McKenzie, J. F.: Wave emission by whistler oscillitons: Application to "coherent lion roars" Geophys. Res. Lett., 29, art.no. 2226, 2002.

Sauer, K., Dubinin, E., and McKenzie, J. F.: Solitons and oscillitons in multi-ion space plasmas, Nonl. Proc. Geophys., 10, 121-130, 2003,

SRef-ID: 1607-7946/npg/2003-10-121.

Verheest, F.: Solitary Alfvén modes in relativistic electron-positron plasmas, Phys. Lett. A, 213, 177-182, 1996.

Verheest, F. and Cattaert, T.: Large amplitude solitary electromagnetic waves in electron-positron plasmas, Phys. Plasmas, 11, 3078-3082, 2004. 